\title{
Finite amplitude evolution of frictionally destabilized abyssal overflows in a stratified ocean
}

\author{
G. E. Swaters \\ Applied Mathematics Institute, Department of Mathematical and \\ Statistical Sciences and Institute for Geophysical Research, \\ University of Alberta, Edmonton, Canada
}

\begin{abstract}
In the immediate vicinity of a deep sill, abyssal ocean overflows can possess current speeds greater the local long internal gravity wave speed with bottom friction and down slope gravitational acceleration playing a dominant role in the dynamics. The parameter regime for the finite amplitude transition to instability is described for marginally unstable super critical frictional abyssal overflows where there is weak coupling between the overflow and gravest-mode internal gravity waves in the overlying water column.
\end{abstract}

\section{Introduction}

The flow of dense water over deep sills is a source point for abyssal ocean currents. These flows, such as, for example, the Denmark Strait Overflow (hereafter DSO, e.g., [1-6]), make an important global-scale contribution to the convective overturing of the oceans. Abyssal currents of this kind are responsible, as well, for deep water replacement in marginal seas (e.g., [7]) and the along slope propagation of cold bottom intensified anomalies (e.g., [8]).

Swaters $[9,10]$ has shown that in the near-inertial regime, super critical overflows can be destabilized by bottom friction. Within the overflow, the instabilities take the form of propagating, growing periodic bores or pulses (and are the rotational analogues of classical roll waves). In the overlying water column the instabilities take the form of amplifying internal gravity waves. For typical DSO parameter values [9], the most unstable mode has a wave length about $45 \mathrm{~km}$, 
propagates prograde with respect to the overflow, has a period about 6 hours, and an $e$-folding growth time about 45 hours.

\section{Governing equations}

The density profile associated with, for example, the Denmark Strait Overflow (see Fig. 5 in [4] and the discussion in [10]) suggests that it is appropriate to consider a $2 \frac{1}{2}$-layer stably stratified abyssal model with variable bottom topography (see Fig. 1). The uppermost layer, which is passive and infinitely deep, is denoted as layer one. The middle, or active upper layer is of finite thickness and is denoted as layer two. The abyssal layer, i.e., the layer immediately above the bottom topography, is denoted as layer 3. The nondimensional equations of motion for the upper layer are given by [10]

$$
\begin{gathered}
\left(\partial_{t}+\varepsilon \gamma^{2} u_{2} \partial_{x}\right) u_{2}=-\eta_{x}+\frac{1}{R_{e}} \partial_{x x} u_{2}, \\
(\eta-h)_{t}+\gamma^{2}\left\{u_{2}\left[1+\varepsilon\left(\eta-h-h_{B}\right)\right]\right\}_{x}=0,
\end{gathered}
$$

and, for the abyssal layer,

$$
\begin{gathered}
\left(\partial_{t}+u_{3} \partial_{x}\right) u_{3}=-p_{x}+\frac{\partial_{x}\left(h \partial_{x} u_{3}\right)}{R_{e} h}-\frac{c_{D}\left|u_{3}\right| u_{3}}{h}, \\
h_{t}+\left(u_{3} h\right)_{x}=0, p=h+h_{B}+\varepsilon \gamma^{2} \eta
\end{gathered}
$$

where $u_{2}, \eta, u_{3}, p$ and $h$ are, respectively, the active upper layer horizontal velocity, the reduced upper layer pressure, the abyssal layer velocity, the reduced abyssal layer pressure and the abyssal layer thickness relative to the height of the bottom topography $h_{B}$.

The Reynolds number $R_{e}$, scaled bottom drag coefficient $c_{D}$, and the parameters $\varepsilon$ and $\gamma$ are given by, respectively,

$$
R_{e} \equiv \frac{L \sqrt{g^{\prime} h_{*}}}{A_{H}}, c_{D} \equiv \frac{c_{D}^{*}}{s^{*}}, \varepsilon \equiv \frac{h_{*}}{H}, \gamma^{2} \equiv \frac{\widetilde{g} H}{g^{\prime} h_{*}},
$$

where $s^{*}$ a representative value for the slope of the bottom topography, $h_{*}$ is a representative value for the thickness of the abyssal layer,

$$
L \equiv \frac{h_{*}}{s^{*}},
$$

the reduced gravities are

$$
\begin{gathered}
g^{\prime}=\frac{g\left(\rho_{3}-\rho_{2}\right)}{\rho_{2}}>0, \\
\widetilde{g}=\frac{g\left(\rho_{2}-\rho_{1}\right)}{\rho_{2}}>0,
\end{gathered}
$$


where

$$
0<\rho_{1}<\rho_{2}<\rho_{3},
$$

and where the horizontal eddy coefficient is $A_{H}$ and the bottom drag coefficient is $c_{D}^{*}$.

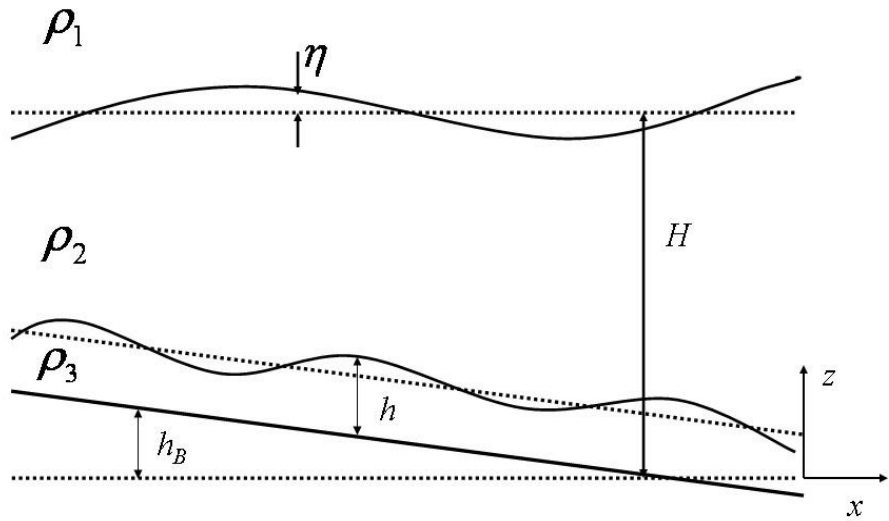

Figure 1: Model geometry used in this paper.

The parameter $\varepsilon$, which is the ratio of the abyssal scale thickness to the overall reference mean depth (and must be less than one), is a measure of the magnitude of the dynamical feedback of the upper layer pressure field back onto the lower layer, and is also a measure of the nonlinearity in the upper layer dynamics. The parameter $\gamma$ is the ratio of the scale long internal gravity wave speeds associated with the dynamically active upper layer to the abyssal layer, respectively.

Oceanographic estimates for the dimensional parameters suggest that (see, e.g., $[5,10-13])$

$$
\left.\begin{array}{c}
\sqrt{g^{\prime} h_{*}} \approx 46 \mathrm{~cm} / \mathrm{s}, L \approx 15 \mathrm{~km}, T \approx 9 \text { hours }, \\
c_{D} \approx 0.25, \varepsilon \approx 0.38, \gamma \approx 2.56, R_{e} \approx 279 .
\end{array}\right\}
$$

It is assumed that $0<\varepsilon \ll 1$, which is the expansion parameter, and that $c_{D}, \gamma$ and $R_{e}$ are formally $O(1)$.

\section{Parameter regime for marginal instability}

The steady abyssal flow solutions which have relevance [5] in the near sill region, and upon which the theory of classical roll waves has been developed, are the "slab" solutions on a linearly sloping bottom (see, e.g., [14-16]) given by

$$
u_{2}=\eta=0, u_{3}=U=\frac{1}{\sqrt{c_{D}}}, h=1, h_{B}=-x .
$$


These uniform flows are equivalent to the "stream tube" solutions, without alongstream variation, which have been used to examine aspects of the dynamics of turbidity and abyssal currents (e.g., [17-20]).

Substitution of the perturbed solution

$$
\left(u_{2}, \eta, u_{3}, h\right) \simeq\left(0,0, \frac{1}{\sqrt{c_{D}}}, 1\right)+\left(\widetilde{u}_{2}, \widetilde{\eta}, \widetilde{u}_{3}, \widetilde{h}\right),
$$

into (1) - (4), leads to the linear stability problem, after dropping the tildes and a little algebra,

$$
\begin{gathered}
\left(\partial_{t}-\frac{\partial_{x x}}{R_{e}}\right)(\eta-h)_{t}-\gamma^{2} \eta_{x x}=0 \\
{\left[\left(\partial_{t}+\frac{\partial_{x}}{\sqrt{c_{D}}}\right)^{2}-\partial_{x x}+\partial_{x}+\left(2 \sqrt{c_{D}}-\partial_{x x}\right)\left(\partial_{t}+\frac{\partial_{x}}{\sqrt{c_{D}}}\right)\right] h=0 .}
\end{gathered}
$$

Assuming a normal mode solution of the form

$$
(h, \eta)=(\widehat{h}, \widehat{\eta}) \exp (i k x+\sigma t)+c . c .
$$

where c.c. means the complex conjugate of the preceding term, leads to the algebraic system, after dropping the carets,

$$
\eta=\delta h,\left(\sigma-\sigma_{+}\right)\left(\sigma-\sigma_{-}\right) h=0,
$$

with

$$
\begin{gathered}
\delta \equiv \frac{\sigma\left(\sigma+k^{2} / R_{e}\right)}{\sigma\left(\sigma+k^{2} / R_{e}\right)+k^{2} \gamma^{2}} \\
\sigma_{ \pm} \equiv-\left(\frac{i k}{\sqrt{c_{D}}}+\sqrt{c_{D}}+\frac{k^{2}}{2 R_{e}}\right) \pm \sqrt{\left(\sqrt{c_{D}}+\frac{k^{2}}{2 R_{e}}\right)^{2}-\left(i k+k^{2}\right)},
\end{gathered}
$$

where the branch cut is taken along the negative real axis. For a nontrivial solution to (12) it follows that $\sigma=\sigma_{ \pm}$.

A mode with a given wave number $k$ will be stable provided

$$
\operatorname{Re}\left(\sigma_{+}\right) \leq 0
$$

i.e.,

$$
\operatorname{Re}\left(\sqrt{\left(\sqrt{c_{D}}+\frac{k^{2}}{2 R_{e}}\right)^{2}-\left(i k+k^{2}\right)}\right) \leq \sqrt{c_{D}}+\frac{k^{2}}{2 R_{e}} .
$$

This can be considerably simplified by introducing the Euler decomposition

$$
\alpha \exp (i \beta)=\left(\sqrt{c_{D}}+\frac{k^{2}}{2 R_{e}}\right)^{2}-k^{2}-i k,
$$


(which serves to define the real numbers $\alpha$ and $\beta$ ) allowing the above stability condition to be re-written in the form

$$
\alpha[1+\cos (\beta)] \leq 2\left(\sqrt{c_{D}}+\frac{k^{2}}{2 R_{e}}\right)^{2},
$$

or, equivalently, after substituting in for $\alpha$ and $\beta$

$$
\left[\left(\sqrt{c_{D}}+\frac{k^{2}}{2 R_{e}}\right)^{2}-k^{2}\right]^{2}+k^{2} \leq\left[\left(\sqrt{c_{D}}+\frac{k^{2}}{2 R_{e}}\right)^{2}+k^{2}\right]^{2},
$$

from which it follows that stability occurs if and only if (for $k \neq 0$, the flow is unconditionally stable for $k=0$ )

$$
\sqrt{c_{D}}+\frac{k^{2}}{2 R_{e}} \geq \frac{1}{2}
$$

In the $R_{e} \rightarrow \infty$ limit, (15) reduces to the classical roll wave stability result

$$
c_{D} \geq \frac{1}{4}
$$

(see, e.g., [14-16]).

Note that (15) implies the existence of a high wave number cutoff if $R_{e}$ is finite. If $R_{e}$ is infinite, the instability problem has an ultraviolet catastrophe, which violates the a priori assumptions for shallow water modelling.

The marginal modes are described by

$$
\begin{gathered}
\sqrt{c_{D}}+\frac{k^{2}}{2 R_{e}}=\frac{1}{2}-\varepsilon \mu \\
\Longrightarrow c_{D}=\widetilde{c}_{D}-2 \varepsilon \mu \sqrt{\widetilde{c}_{D}}+\varepsilon^{2} \mu^{2},
\end{gathered}
$$

where

$$
\widetilde{c}_{D} \equiv \frac{\left(1-k^{2} / R_{e}\right)^{2}}{4},
$$

is the critical value for $c_{D}$ as a function of $k$ and $R_{e}$. The parameter $\mu \simeq O(1)$ measures the super or subcriticality. Substitution of (16) into (14) implies

$$
\begin{array}{r}
\sigma_{+}=-\frac{1}{2}+\varepsilon \mu-\frac{i k}{\sqrt{c_{D}}}+\sqrt{\frac{(1-2 i k)^{2}}{4}-\varepsilon \mu+\varepsilon^{2} \mu^{2}} \simeq \frac{4 \varepsilon \mu k^{2}}{\left(1+4 k^{2}\right)} \\
-i k\left\{\frac{3-k^{2} / R_{e}}{\left(1-k^{2} / R_{e}\right)}+\frac{2 \varepsilon \mu\left[\left(1-k^{2} / R_{e}\right)^{2}+2\left(1+4 k^{2}\right)\right]}{\left(1+4 k^{2}\right)\left(1-k^{2} / R_{e}\right)^{2}}\right\}+O\left(\varepsilon^{2}\right),
\end{array}
$$

so that the growth rate of the marginal mode will be $O(\varepsilon)$. Marginally unstable (stable) modes correspond to $\mu>(<) 0$, respectively. A detailed description of the nonlinear evolution of the amplitudes of the marginally unstable modes, assuming $0<\varepsilon<<1$ in (17), will be described elsewhere. 


\section{Conclusions}

The parameter regime for the weakly nonlinear marginal destabilization of frictional supercritical abyssal overflows with weak coupling between the overflow and gravest-mode internal gravity waves in the overlying water column has been described. Necessary and sufficient stability conditions have been derived. It has been shown that in the limit of infinite Reynolds number the stability conditions reduce the known classical results. The inclusion of a finite Reynolds number removes the ultraviolet catastrophe known to exist in the stability problem when turbulent horizontal mixing is not present, violating the a priori assumptions for modelling with the shallow water equations.

\section{Acknowledgement}

Preparation of this extended abstract was supported in part by the Natural Sciences and Engineering Research Council of Canada.

\section{References}

[1] Worthington, L. V., An attempt to measure the volume transport of Norwegian Sea overflow water through the Denmark Strait. Deep-Sea Res., 1969, 16, 421-432.

[2] Dickson, R. R. and Brown, J., The production of North Atlantic Deep Water: Sources, rates, and pathways. J. Geophys. Res., 1994, 99, 12319-12341.

[3] Käse, R. H. and Oschlies, A., Flow through Denmark Strait. J. Geophys. Res., 2000, 105, 28527-28546.

[4] Girton, J. B. and Sanford, T. B., Synoptic sections of the Denmark Strait overflow. Geophys. Res. Lett., 2001, 28, 1619-1622.

[5] Girton, J. B. and Sanford, T. B., Descent and modification of the Denmark Strait overflow. J. Phys. Oceanogr., 2003, 33, 1351-1364.

[6] Jungclaus, J. H., Hauser, J. and Käse, R. H., Cyclogenesis in the Denmark Strait Overflow plume. J. Phys. Oceanogr., 2001, 31, 3214-3228.

[7] LeBlond, P. H., Ma, H., Doherty, F. and Pond, S., Deep and intermediate water replacement in the Strait of Georgia. Atmos.-Ocean, 1991, 29, 288312.

[8] Houghton, R. W., Schlitz, R., Beardsley, R. C., Butman, B. and Chamberlin, J. L., The middle Atlantic bight cold pool: Evolution of the temperature structure during summer 1979. J. Phys. Oceanogr., 1982, 12, 1019-1029.

[9] Swaters, G. E., Baroclinic characteristics of frictionally destabilized abyssal overflows. J. Fluid Mech., 2003, 489, 349-379.

[10] Swaters, G. E., On the frictional destabilization of abyssal overflows dynamically coupled to internal gravity waves. Geophys. Astrophys. Fluid Dynamics, in press, 2006. 
[11] Spall, M. A. and Price, J. F., Mesoscale variability in Denmark Strait: The PV outflow hypothesis. J. Phys. Oceanogr., 1998, 28, 1598-1623.

[12] Reszka, M. K., Swaters, G. E. and Sutherland, B. R., Instability of abyssal currents in a continuously stratified ocean with bottom topography. J. Phys. Oceanogr., 2002, 32, 3528-3550.

[13] Käse, R. H., Girton, J. B. and Sanford, T. B., Structure and variability of the Denmark Strait overflow: Model and observations. J. Geophys. Res., 2003, 108 (C6), 3181 10.1029/2002JC001548.

[14] Jeffreys, H., The flow of water in an inclined channel of rectangular bottom. Phil. Mag., 1925, 49, 793-807.

[15] Whitham, G. B., Linear and Nonlinear Waves, 1974 (Wiley: New York, Chichester, Brisbane, Toronto).

[16] Baines, P. G., Topographic Effects in Stratified Flows, 1995 (Cambridge University Press: Cambridge, New York, Melbourne).

[17] Smith, P. C., A streamtube model for bottom boundary currents in the ocean. Deep-Sea Res., 1975, 22, 853-873.

[18] Killworth, P. D., Mixing on the Weddell Sea continental slope. Deep-Sea Res., 1977, 24, 427-448.

[19] Price, J. F. and Baringer, O. M., Outflows and deep water production by marginal seas. Progr. Oceanogr., 1994, 33, 161-200.

[20] Emms, P. W., A streamtube model of rotating turbidity currents. J. Mar. Res., 1998, 56, 41-74. 